\title{
Propriété, biens publics mondiaux, bien(s) commun(s): Une lecture des concepts économiques
}

\section{Jérôme Ballet}

\section{OpenEdition}

Journals

Édition électronique

URL : http://journals.openedition.org/developpementdurable/5553

DOI : 10.4000/developpementdurable.5553

ISSN : 1772-9971

Éditeur

Association DD\&T

Référence électronique

Jérôme Ballet, «Propriété, biens publics mondiaux, bien(s) commun(s) : Une lecture des concepts économiques », Développement durable et territoires [En ligne], Dossier 10 | 2008, mis en ligne le 07 mars 2008, consulté le 19 avril 2019. URL : http://journals.openedition.org/ developpementdurable/5553 ; DOI : 10.4000/developpementdurable.5553

Ce document a été généré automatiquement le 19 avril 2019.

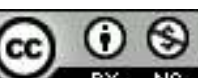

Développement Durable et Territoires est mis à disposition selon les termes de la licence Creative Commons Attribution - Pas d'Utilisation Commerciale 4.0 International. 


\title{
Propriété, biens publics mondiaux, bien(s) commun(s) : Une lecture des concepts économiques
}

\author{
Jérôme Ballet
}

1 La référence de plus en plus récurrente ces dernières années au concept de bien public mondial pour aborder les questions relatives aux problèmes affectant le monde dans son ensemble ou au moins une large part de sa population s'inscrit dans une volonté de caractériser de manière relativement homogène une série de phénomènes variés: le changement climatique, les risques de propagation des maladies contagieuses, l'instabilité financière, l'insécurité, etc. ${ }^{1}$

2 Or le débat qui s'ouvre avec cette nouvelle notion renvoie à deux questions traitées dans la théorie économique. La première est celle du régime de propriété adapté pour répondre à de tels phénomènes. La seconde concerne la lignée de la notion de bien public mondial vis-à-vis de celle de bien public présente depuis longtemps dans la théorie économique. Cet article s'interroge donc d'abord sur ces deux questions et propose une lecture des concepts afin d'éclaircir le débat.

3 La filiation de la notion de bien public mondial avec celle de bien public est partielle. D'une part, tout en visant à conserver les caractéristiques usuelles qui définissent un bien public (la non-rivalité et la non-exclusion), elle procède d'un double dépassement du champ d'analyse : par la dimension temporelle et la dimension territoriale. Cependant, la notion de bien public mondial, ou plus exactement ce qui est rangé derrière cette notion, recèle une certaine ambiguïté puisque certains biens tels des ressources naturelles seront parfois classés dans cette catégorie alors qu'ils ne possèdent pas ces deux caractéristiques. D'autre part, la notion de bien public mondial semble renvoyer au moins implicitement à la question de l'équité. Mais alors elle propose un déplacement relatif de l'analyse économique.

4 En effet, la problématique traditionnelle de la théorie économique consiste à caractériser le système de droits de propriété le mieux adapté pour assurer de manière la plus efficiente possible la production ou la gestion des biens. La notion de bien public mondial 
n'échappe pas à cette problématique, mais en même temps introduit une ambiguïté en insérant implicitement la problématique de l'équité dans le débat. Certains biens publics mondiaux apparaissent alors assez loin de la conception conventionnelle des biens publics, ce qui laisse penser que la catégorisation des biens publics mondiaux est dans une certaine mesure abusive en intégrant des éléments qui ne présentent pas nécessairement les caractéristiques de biens publics. Cette intégration est certainement due à la volonté de faire pencher le débat vers la problématique de l'équité. Mais dans ce cas, il conviendrait d'afficher plus clairement l'importance de ce critère, de sorte que le débat sur le régime de propriété adapté ne se réfère plus uniquement au critère d'efficience. Un parallèle entre les biens publics mondiaux et le Bien commun, autre notion qui apparaît régulièrement récemment, s'avère alors porteur de sens.

1. Retour sur quelques notions et leur rapport avec les droits de propriété

5 La référence en théorie économique aux droits de propriété renvoie à la théorie du même nom. Cette théorie souligne la variété de configuration des systèmes de droits de propriété selon les contextes. Au-delà de la variété, une catégorisation des régimes de droits est souvent proposée. Un des enseignements clés de cette approche est qu'en présence d'externalités un système de droits de propriété privée permet leur internalisation. Autrement dit, pour réduire une externalité il suffit de créer un nouveau marché reposant sur un système de droits ${ }^{2}$. Cependant, le régime de propriété adapté à chaque situation dépend en bonne partie de la nature des biens en jeu. À cet égard les débats sur les régimes de propriété adaptés en présence d'externalité, de biens publics et de ressources communes sont conséquents. Cette première section présente un rappel des principales notions, des présupposés de la théorie des droits de propriété et certaines remises en cause.

1.1. Théorie des droits de propriété et structure des droits de propriété

6 La théorie des droits de propriété se situe dans la perspective de compréhension des différentes formes d'institutions économiques. En effet, définir un système de droits de propriété revient en fait à élaborer une forme d'institution. La notion de droit de propriété retenue dans cette approche est très large et ne se limite pas à une délimitation juridique. Un droit de propriété comprend également les coutumes, les règles et normes qui définissent et délimitent les usages « autorisés » et/ou "légitimes» des actifs. Un droit de propriété est de manière générale un droit socialement légitime à choisir les usages d'un bien économique. Ainsi selon Barzel (1989) un droit de propriété consiste en la capacité ou au pouvoir de consommer un actif, d'en obtenir un revenu ou de l'aliéner. La théorie des droits de propriété conçoit donc la nature du droit en relation avec la capacité d'usage des actifs. Plus un individu aura une capacité d'usage forte sur un actif plus il pourra être considéré comme le propriétaire de cet actif.

7 La théorie des droits de propriété est par conséquent une théorie générale des institutions et des relations sociales dans laquelle l'ensemble des relations sont médiatisées par des droits sur des actifs et plus généralement où les relations sociales découlent de relations entre objets (Alchian 1969).

8 La garantie de droits sur les biens et leur aliénabilité devient alors une condition de fonctionnement d'une économie décentralisée (Alchian 1987). L'opposition entre le marché et la firme en raison de coûts de transaction (Coase 1937) n'est pas alors une opposition de nature, mais une opposition d'arrangements institutionnels reflétant un système de droits de propriété. Si en raison des coûts de fonctionnements du marché, en particulier l'existence de coûts de transaction, la firme peut devenir un mode alternatif 
de transaction (Coase 1937), la firme est elle-même un ensemble de contrats (Alchian et Demsetz 1972) associé à des droits de propriété ou définissant une structure des droits de propriété (Ricketts 1987). Comme le souligne Coase (1960), les ressources d'une firme ne sont pas des ressources physiques mais des droits de propriété (sur ces ressources physiques). L'existence de coûts de transaction implique donc que la firme, en tant que système de droits de propriété, s'avère plus efficiente que le marché. Autrement dit, les coûts de transaction justifient que l'organisation des droits de propriété se fasse sous la forme d'une firme plutôt que d'un marché. Le système de droits de propriété est par conséquent dépendant des coûts de transaction.

Le choix d'une forme organisationnelle ou système de droits de propriété ne signifie pas que les droits de propriété sont détenus par une seule personne, mais au contraire que ces droits sont partitionnables de sorte que plusieurs individus pourront avoir des droits sur un même actif (par exemple les managers et les actionnaires).

10 Une forme d'organisation est alors le reflet de la manière dont les droits de propriété sur des actifs sont délimités et affectés aux individus. Le système de droits de propriété ainsi constitué génère une certaine efficacité comparée à d'autres formes de systèmes de droits de propriété.

11 Le résultat essentiel de la théorie des droits de propriété est par conséquent que des structures de droits de propriété émergent en réponse à des problèmes d'allocation des ressources. Cette structure affecte en retour les comportements et les systèmes économiques (Coase 1960, Furubotn et Pejovitch 1972). Ainsi, selon Alchian et Demsetz (1972) la firme capitaliste classique, c'est-à-dire la société par action, et le système de droits qui la définit se sont imposés historiquement parce qu'ils permettent de résoudre les problèmes d'information imparfaite et de risque moral que l'on trouve dans les productions en équipe et ce de manière plus efficiente que ne le ferait un arrangement contractuel décentralisé.

12 Cette optique ouvre la voie à une lecture comparée des coûts et avantages des différentes structure de droits de propriété (Coase 1960) et dans une perspective évolutionniste (Anderson et Hill 1975, North et Thomas 1977, Liebecap 1989, North 1990) offre une grille de lecture sur les processus permettant d'analyser les choix institutionnels, les intérêts et les conflits d'intérêts des parties contractantes et la persistance de régimes de droits de propriété inefficients, ainsi qu'une analyse de la dégradation des droits de propriété (Amann 1999).

\subsection{Les régimes de propriété}

13 Au-delà des variations importantes des structures de droits de propriété, une classification en types de régimes permet de regrouper l'ensemble des variations. Il est usuel de distinguer entre les régimes d'accès libre, de propriété commune, de propriété d'Etat et de propriété privée (Seabright 1993).

14 1) Le régime d'accès libre reflète l'absence de droit et de possibilité de contrôle. Par exemple les ressources marines dans les eaux internationales, l'air ou l'atmosphère sont librement accessibles, sans droit. L'accès est donc libre et non régulé ce qui provoque potentiellement une sur-utilisation et une exploitation abusive de la ressource. La pêche surabondante dans les eaux internationales et les pollutions transfrontalières illustrent bien ce problème (Mäler 1990). Un tel régime renvoie alors à ce qu'il est convenu d'appeler la tragédie des communaux (Hardin 1968). ${ }^{3}$. Un tel régime de propriété est assez rare sur les ressources naturelles terrestres. Cependant, des situations analogues se 
rencontrent dès lors que les droits sur les ressources sont seulement nominaux et que l'utilisation des ressources est de fait libre. Par exemple les parcs nationaux ou les réserves sur lesquels le contrôle effectif des droits est impossible ou non mis en œuvre s'apparentent à ce régime, d'autant plus que les populations locales estiment posséder un droit légitime, par exemple en raison de coutumes et de l'histoire, à l'utilisation de la ressource (Heltberg 2001).

2) Le régime de propriété commune renvoie à un système de possession communal où l'accès à la ressource est défini selon des règles régissant cette ressource et supposant que l'accès est restreint aux membres de la communauté. Ce régime de propriété peut s'appuyer sur différents types de communautés : la tribu, le village, le clan ou le lignage, la communauté d'usage, la coopérative, la municipalité ou le gouvernement local. Il concerne de nombreuses ressources comme les forêts communes, les eaux territoriales pour la pêche, les systèmes d'irrigation, etc. Ce type de régime est confronté comme le précédent à un risque de passager clandestin si le contrôle et l'implémentation des droits ne sont pas effectifs. Baland et Platteau (1996) distinguent pour cette raison les propriétés communes régulées, pour lesquelles un ensemble de règles de conservation et d'utilisation de la ressource est clairement établi, des propriétés communes non régulées, pour lesquelles aucune règle ne limite l'utilisation pour les membres. Autrement dit les ressources sous le régime de propriété commune non régulée sont utilisables par tous les membres de la communauté, et ceux là seulement, mais sans limites ou restrictions sur l'utilisation par ces membres. A l'inverse, les ressources sous le régime de propriété commune régulée sont non seulement restreintes d'accès aux membres de la communauté mais leur utilisation par ces membres est également sujette à des restrictions définies par les règles de la communauté. Ainsi, les ressources soumises au régime de propriété commune non régulée sont confrontées au risque de sur-utilisation en raison de comportements opportunistes de la part de certains membres.

3) Le régime de propriété d'Etat est un régime dans lequel les ressources sont en possession de l'Etat et sous son contrôle concernant aussi bien l'accès que les règles de conservation. Cependant, quand l'Etat s'avère incapable d'implémenter et contrôler effectivement l'accès et l'utilisation des ressources, alors celles-ci deviennent de fait soit en accès libre, soit accaparées à titre privé. Les réserves ou les forêts classées se retrouvent souvent dans ce type de situation. L'incapacité à contrôler la ressource implique bien souvent que des populations avoisinantes en utilisent des parcelles pour la culture.

17 4) Le régime de propriété privée concerne le cas de ressources dont les droits sont détenus par des individus. Lorsque les droits de propriété privée sont parfaits, c'est-à-dire lorsqu'ils sont complets, sécurisés, transférables et permettent au possesseur la pleine utilisation, alors un tel régime associé avec un système de marchés complet permet d'atteindre un optimum au sens de Pareto. En revanche, dès lors qu'un tel régime s'avère incomplet c'est-à-dire que les droits de jure ne sont pas effectifs de facto, en particulier que les coûts de transaction sont élevés, son efficience est remise en cause ${ }^{4}$.

\subsection{Externalités, Biens Publics et Ressources communes}

Cette sous-section propose une lecture des concepts d'externalités, biens publics et ressources communes à l'aune des résultats de la théorie des droits de propriété, avec la relative partialité de cette théorie, avant de présenter dans la sous-section suivante une remise en cause de ces résultats. Un des résultats, ou des présupposés, de la théorie des droits de propriété est qu'un système adéquat de droits de propriété permet 
d'internaliser les externalités (Demsetz 1967). En particulier, en ce domaine, la théorie des droits de propriété postule la supériorité des droits de propriété privée sur les propriétés collectives (Furubotn et Pejovitch 1972). Un droit échangeable, donc un droit ayant les caractéristiques d'un droit de propriété privée, assure l'allocation optimale des ressources en permettant d'internaliser les externalités en raison des mécanismes incitatifs générés par ce droit. En effet, un droit permet de tirer un avantage pour soi ou pour d'autres de la ressource sur laquelle l'individu dispose du droit (ou éventuellement de se léser soi-même ou un autre). Par exemple, un droit à polluer incite à moins polluer afin de pouvoir vendre son droit et en tirer un bénéfice. En ce sens, alors qu'une taxe est pénalisante si le pays ne respecte pas le niveau de pollution admis, en particulier si elle est progressive avec le niveau de pollution, un droit à polluer assure un gain, grâce à la revente du droit, si le pays émet une pollution en deçà de la norme fixée. Le système de droits à polluer permet donc d'une part plus de souplesse que la taxe, d'autre part assure des revenus supplémentaires pour ceux qui sont les plus vertueux ${ }^{5}$.

Or les externalités présentent des traits similaires aux biens publics. Dans les deux cas il y a indivisibilité de la consommation. Rappelons qu'un bien public pur se définit par les caractéristiques de non exclusion et de non rivalité de la consommation. Autrement dit, personne ne peut être exclu de la consommation et la consommation par une personne ne réduit pas la consommation d'une autre personne. La différence entre externalité et bien public pur réside dans l'étendue de l'indivisibilité. «La différence est que l'externalité, qui introduit une indivisibilité certaine, se greffe sur un bien qui reste divisible en ce sens qu'il a des acheteurs individuels (ou consommateurs directs) qui en règlent souverainement le débit. En revanche la consommation du bien collectif est totalement indivisible. C'est en quelque sorte un bien qui n'aurait que des externalités. » (Benard 1985, p.120).

D'une certaine manière, un système de droits de propriété adapté, particulièrement un système de droits de propriété privée, résout le problème des externalités en modifiant les conditions de l'action collective, soit en incitant à participer à la production d'une externalité positive, soit en incitant à participer à la réduction d'une externalité négative. Il élimine de ce fait les comportements de passagers clandestins qui sont eux aussi au cœur du problème de fourniture des biens publics purs. Ainsi la pollution est une externalité tandis que l'air pur est un bien public pur. La mise en place de droits à polluer vise à internaliser l'externalité -pollution et permet ainsi de conserver le bien public qu'est l'air pur. Il s'agit cependant là d'une simple incitation et non d'une solution miracle puisque si tout le monde utilise son droit à polluer l'air sera de fait pollué et le bien public air pur dégradé.

21 Dans ce cadre, il convient de maintenir la distinction entre externalité et bien public. Un bien public est par nature particulièrement susceptible d'être soumis à un régime d'accès libre puisque personne ne peut être exclu de sa consommation 6 . Or un tel régime, comme souligné précédemment, conduit à une situation de sur-utilisation de la ressource. Et en règle générale, la théorie économique souligne que la fourniture d'un bien public, si elle est laissée à l'initiative privée, est sous-optimale. Le comportement de passager clandestin est la règle et chacun tente de bénéficier de la ressource ou du bien sans contribuer à sa fourniture ${ }^{7}$. Par ailleurs, dans de nombreux cas, le bien public est dégradé en raison des comportements individuels. L'émission de pollution dégrade le bien public " air pur ». Autrement dit, si le fait de respirer ne réduit pas le volume d'air disponible pour les autres personnes (le bien est non rival), en revanche certains comportements 
affectent la qualité de ce bien. Or nous retrouvons là le phénomène d'externalité. Il devient alors possible de résoudre le problème de la dégradation du bien public par la définition de droits de propriété, tels les droits à polluer.

Il faut en fait distinguer deux types de biens publics. D'une part les biens publics immédiatement disponibles aux individus sans qu'ils aient une action quelconque à mener. C'est le cas de l'air, des eaux internationales, etc., donc un ensemble de ressources librement disponibles. Il s'agit de biens publics naturels (il faut distinguer ces biens publics naturels des ressources communes, voir plus loin). Dans ce cas, le problème n'est pas celui de la fourniture de ce bien puisqu'il préexiste à l'action humaine, il est celui de sa conservation ou de sa dégradation. D'autre part les biens publics qui doivent être fournis par l'action humaine, par exemple l'éclairage public la nuit. Dans ce cas, le problème n'est plus seulement celui de son utilisation mais celui de sa production ou de sa fourniture. Or ici un système de droits de propriété privée ne permet pas une fourniture optimale. Dans ce cas de figure, il faut établir soit un système de propriété commune, soit un système de propriété d'Etat, ou un système de propriété privée par un monopole sous tutelle de l'Etat ${ }^{8}$. En effet, en raison de risques de comportement de passager clandestin, la production de ce type de bien suppose que l'on ait recours à un financement collectif, généralement par la taxation indépendamment de l'utilisation. Cela n'exclut pas bien sûr certains comportements de dégradation du bien, par exemple le fait que certains individus cassent les lampadaires publics. Néanmoins, une utilisation normale du bien conduit à une stabilité de sa qualité.

Enfin, le terme ressource commune est consacré pour les questions relatives aux ressources environnementales. Ces ressources environnementales sont caractérisées par la non exclusion mais une certaine rivalité dans l'utilisation. La tragédie des communaux (Hardin 1968) reflète bien ce problème. La sur-utilisation des communaux laissés en accès libre provoque la dégradation de la ressource. Il s'agit bien là d'un cas différent de l'air. L'air se dégrade non pas en raison d'un comportement de passager clandestin mais en raison d'une externalité, i.e. la pollution, donc d'une activité autre que le fait de respirer. Par contre, la dégradation des forêts est le résultat d'une utilisation directe de ces forêts sans respect pour le temps de reboisement, etc. Il a donc une différence de nature quant aux modalités de dégradations. Le terme ressource commune est réservé au second cas (les forêts et autres ressources du même type) et le terme bien public utilisé pour le premier cas (l'air et les biens naturels du même genre). Mais dans ce cas comme dans les autres, la théorie des droits de propriété conclut à la supériorité du régime de propriété privée puisqu'il fournit une garantie sur l'utilisation future et réduit donc le comportement de sur-utilisation présent pour un étalement dans le temps de la ressource compatible avec une meilleure gestion. Cette optique est en bonne partie à l'origine des politiques de définition et de redécoupage des droits sur les ressources dans certains pays en développement (voir par exemple pour une première approche Heltberg 2002).

1.4. L'efficience des droits de propriété privée en question

La présentation précédente, conformément à la théorie des droits de propriété, laisse penser que dans de nombreux cas le régime de propriété privée est adapté pour résoudre les problèmes d'efficience. Hormis les biens publics nécessitant d'être produits ${ }^{9}$, toutes les autres catégories pourraient trouver avantage à passer sous le régime de propriété privée (cf. tableau synthétique 1), notamment par la mise en place de mécanismes de marché adaptés. 
Tableau 1. Types de biens et régimes de propriété

\begin{tabular}{|c|c|c|c|}
\hline Types de biens & Caractéristiques des biens & Exemple de bien & $\begin{array}{l}\text { Régimes de propriété } \\
\text { "adaptés" selon la } \\
\text { théorie des droits de } \\
\text { propriété }\end{array}$ \\
\hline Bien privé & Rivalité et exclusion & Automobile & Propriété privée \\
\hline Bien à péage & 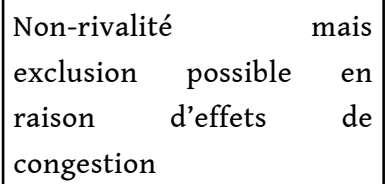 & $\begin{array}{l}\text { Piscine, } \\
\text { autoroute }\end{array}$ & Propriété privée \\
\hline $\begin{array}{l}\text { Ressources } \\
\text { communes }\end{array}$ & Rivalité et non exclusion & $\begin{array}{l}\text { Forêt } \\
\text { communautaire }\end{array}$ & Propriété privée \\
\hline $\begin{array}{lr}\text { Bien } & \text { public } \\
\text { disponible } & \text { sans } \\
\text { production-naturel }\end{array}$ & $\begin{array}{l}\text { Non-rivalité et non } \\
\text { exclusion }\end{array}$ & Air & $\begin{array}{l}\text { propriété privée par la } \\
\text { création de marchés }\end{array}$ \\
\hline $\begin{array}{ll}\text { Bien } & \text { public } \\
\text { nécessitant } & \text { d'être } \\
\text { produit } & \end{array}$ & $\begin{array}{l}\text { Non-rivalité et non } \\
\text { exclusion }\end{array}$ & Eclairage public & $\begin{array}{l}\text { Propriété commune ou } \\
\text { propriété d'Etat }\end{array}$ \\
\hline
\end{tabular}

Sans proposer une revue exhaustive des critiques adressées à cette théorie, nous relevons quelques remises en cause sérieuses des résultats qu'elle propose. Premièrement, cette grille de lecture historique des choix institutionnels a été régulièrement critiquée pour le biais libéral qu'elle implique, défendu au prix d'un détournement des faits historiques (Runge et Bromley 1979, Tartarin 1982, Bromley 1989, Boisvert et al. 2004).

Deuxièmement, si on exclut le cas des biens publics nécessitant d'être produits, la supériorité de la propriété privée pour les biens publics naturels et les ressources communes repose sur la capacité de ce régime à favoriser l'internalisation des externalités et réduire les comportements de passager clandestin. La propriété privée est supposée résoudre ces deux problèmes. Or un tel résultat a été largement remis en cause. Larson et Bromley (1990) soulignent que l'efficience supposée de la propriété privée repose sur deux axiomes : l'axiome de composition et l'axiome d'autorité. Selon l'axiome de composition le contrôle complet de la ressource doit être dévolu à un groupe bien défini pour une utilisation efficiente. Selon l'axiome d'autorité, ce groupe doit agir avec un objectif unifié. De fait, la localisation de ces deux conditions en une seule personne détentrice de droits de propriété respecte ces deux axiomes et induit une efficience du régime de propriété privée. Néanmoins, d'une part on ne peut en déduire que d'autres régimes de propriété sont moins efficients et de nombreuses illustrations et argumentations ont fait valoir la relative efficience de la gestion en commun des ressources (de los Reyes et Jopillo 1986, Cernea 1987, Wade 1988, Ostrom 1990, Uphoff 1992, Pretty et al. 1995, Röling et Wagemakers 1997, Heltberg 2002, parmi bien d'autres) ${ }^{10}$, d'autre part le respect de ces deux axiomes (et donc le régime de propriété privée) ne 
suffit pas à garantir une gestion socialement efficiente des ressources, notamment en raison d'une forte préférence pour le présent liée à la contrainte de revenu des populations pauvres (Larson et Bromley 1990).

Troisièmement, la théorie des droits de propriété, prise dans un sens évolutionniste (ce qui semble être généralement le cas), s'avère en fait inadaptée pour évaluer l'efficience de deux systèmes différents de propriété (Tartarin 1987). En effet, si l'efficience renvoie au critère de Pareto, ce dernier permet simplement de classer différents états d'un même système (défini par les mêmes biens, technologies, préférences et droits de propriété) et non différents systèmes. Autrement dit «le critère de Pareto permettrait donc une comparaison intrasystémique mais pas intersystémique des états économiques " (Tartarin 1987, p. 1138). Cette approche renvoie en fait à une assimilation abusive du critère d'efficience avec celui d'échange volontaire puisque l'on suppose qu'à partir du moment où les agents décident d'échanger, c'est qu'ils en tirent un avantage (et donc que l'on se trouve sur un état Pareto supérieur au précédent). Mais il s'agit bien là d'un abus puisque cet argument n'est valable que dans un système donné. Dès lors qu'il y a modification des droits de propriété, le système change également et il n'est plus possible d'utiliser le critère de Pareto puisque se produit non pas un déplacement vers un état Pareto supérieur mais une modification de l'ensemble des possibilités d'optimum.

Quatrièmement, en suivant Tartarin (1987) nous pouvons dire que deux régimes de propriété quelconques voient leur efficacité relative dépendre de la répartition des ressources entre agents, en particulier de l'inégalité des droits, de sorte qu'il ne devient plus possible d'affirmer la supériorité d'un régime sur l'autre. On ne peut ainsi conclure à l'efficience relative d'un marché de droits à polluer par rapport à un autre régime de propriété sans poser avant tout la question de la distribution des droits sur ce marché. De ce point de vue, l'efficience est conditionnée par la répartition des droits, ce qui suppose une analyse de l'équité au préalable ou au moins en complément de l'utilisation du critère d'efficience. Or, il semble que ce soit un des enjeux centraux proposés avec les nouvelles approches des biens publics, en particulier celle de bien public mondial.

2. La notion de bien public mondial

Récemment, la notion de bien public mondial s'est développée. Elle retranscrit la préoccupation de plus en plus forte vis-à-vis de problèmes globaux tels que le changement climatique, l'insécurité, le développement de pandémies au niveau mondial comme le VIH/sida, etc., dans un contexte de mondialisation des économies. Cette section présente cette notion et discute de sa pertinence.

2.1. Du bien public au bien public mondial

Les biens publics, à l'image des luminaires sur les bords des routes ou des phares, sont une problématique classique en théorie économique. La mondialisation, mais aussi l'apparition de nouvelles problématiques, comme celle du changement climatique, ont fait émerger une nouvelle thématique autour des biens publics mondiaux. Cette problématique n'est cependant pas tout à fait nouvelle. Elle prolonge la volonté de certains économistes d'élargir le spectre de la notion de bien public, par exemple avec l'inclusion de la stabilité, la régulation ou la redistribution (Cooper 1977), voire le nationalisme (Breton 1964) mais surtout avec celle de bien public international proposée par Kindleberger (1986). Ce dernier ouvre directement la question de la fourniture de biens publics internationaux en l'absence de gouvernement international. Dans ce cadre, il constate que les biens publics internationaux sont généralement fournis si une puissance, un Etat, hégémonique leader favorise cette fourniture, que ce soit par pur 
intérêt ou en raison de l'existence de normes, règles et procédures de décision à partir desquelles les acteurs internationaux convergent sur un problème spécifique.

31 Cependant, la mondialisation, de par ses caractéristiques (voir par exemple Serfati 2003), ouvre considérablement et avec urgence le débat. Ainsi, « gérer la mondialisation impose de comprendre et d'organiser la fourniture des biens publics mondiaux de façon à ce qu'ils puissent bénéficier à toutes les composantes du public mondial.» (Kaul et al. 2003, p.2). En particulier parce que «la gestion de la mondialisation dépend dans une large mesure de la fourniture de biens publics mondiaux» (Ibid, p.3). Ce que sera la mondialisation et les bénéfices que tous les agents en tireront dépendent dans cette perspective de la capacité à gérer les biens publics mondiaux. Si certains biens sont mondiaux par nature, comme la couche d'ozone, d'autres sont mondialisés ou peuvent être mondialisés de manière intentionnelle, par exemple la santé publique.

Suivant Kaul et al. (1999, p. 16), les biens publics mondiaux ont les caractéristiques suivantes :

- ils bénéficient à plus d'un groupe de pays,

- ils ne discriminent pas entre les groupes de population ou ensembles de générations, présents ou futurs.

- Anand (2004, p. 216) précise cette définition à partir de trois critères :

- ils couvrent plus d'un groupe de pays,

- ils bénéficient, non seulement à un large spectre de pays, mais aussi à un large spectre de la population globale,

- ils répondent aux besoins des générations présentes sans entraver ou entamer ceux des générations futures.

Ce qui caractérise les biens publics mondiaux par rapport aux biens publics est donc l'étendue de leur champ de validité. Ainsi, certains biens publics nationaux peuvent être considérés comme des biens privés au niveau mondial. Kaul et Mendoza (2003) présentent une typologie des catégories de tels biens (cf. figure).

Généralement le degré de pureté des biens publics mondiaux permet d'en distinguer trois grandes catégories (Kaul et al. 1999, p. 453) :

- les biens publics mondiaux naturels (par exemple la couche d'ozone ou la stabilité climatique),

- les biens publics mondiaux fabriqués par l'homme (par exemple les connaissances scientifiques et pratiques, les normes et principes, et l'héritage culturel),

- les résultats des politiques globales (par exemple la paix, la santé ou la stabilité financière). 


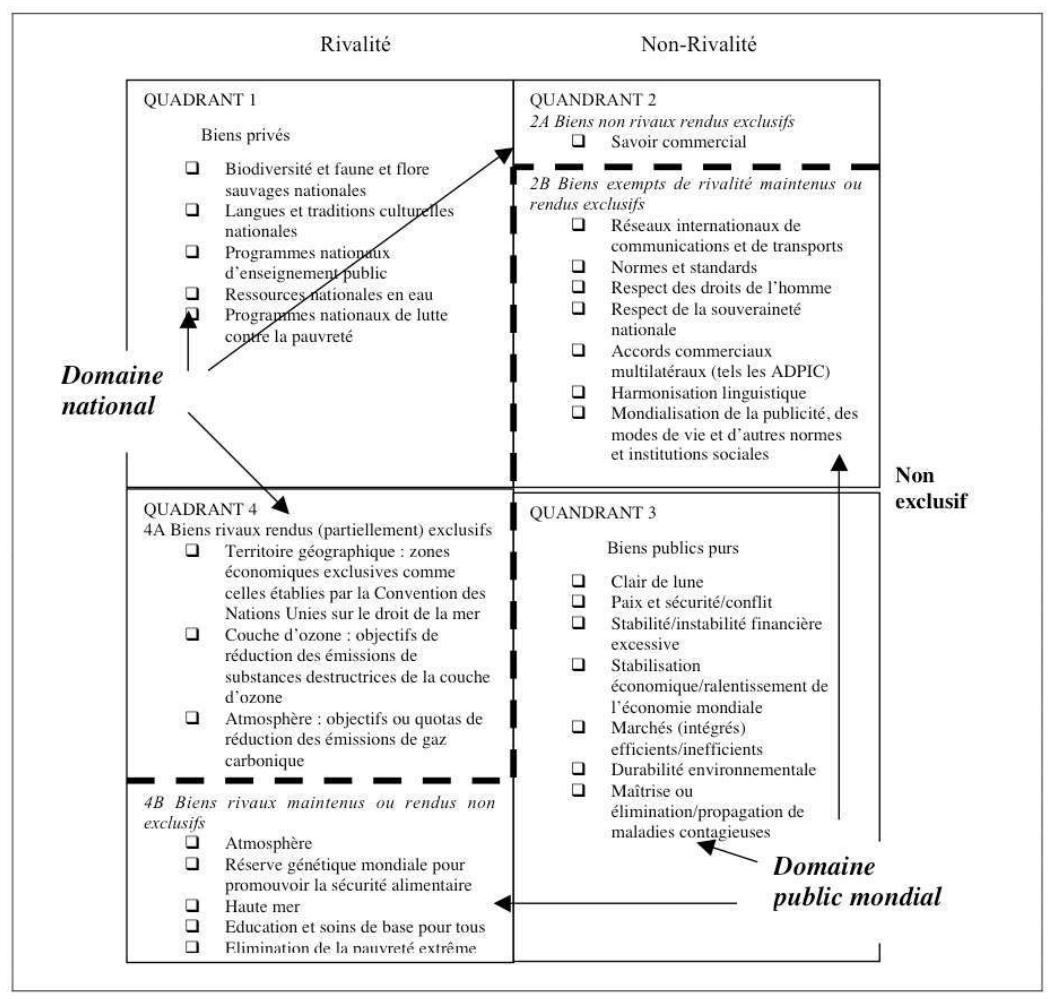

Source : Kaul et al. (2003)

\subsection{Une catégorisation problématique}

Cette typologie des biens publics mondiaux paraît relativement insatisfaisante. Trois remarques principales peuvent être faites. Premièrement, la troisième catégorie (i.e. les résultats des politiques globales) ne se distingue pas toujours de la première catégorie (les biens publics mondiaux naturels). Par exemple la couche d'ozone est certes donnée naturellement, mais les politiques mises en œuvre ou non peuvent aussi modifier son état. Des politiques globales sont nécessaires pour réduire les externalités qui portent atteinte à la qualité du bien. Inversement, la paix peut être considérée comme une donnée dégradée par des comportements prédateurs. Les deux catégories renvoient à la problématique des externalités.

Deuxièmement, la seconde catégorie (les biens publics mondiaux fabriqués par l'homme) est elle aussi problématique. Deneulin et Townsend (2007) soulignent que la typologie proposée par Kaul et al. (1999) est inadaptée parce qu'elle provoque une confusion entre bien commun et bien public. En particulier le fait de considérer l'héritage culturel comme un bien public constitue une erreur d'interprétation puisque ce type de bien est à ranger dans la catégorie des biens communs. À l'inverse du bien public dont la consommation et la production sont séparables, les biens communs sont caractérisés par une production et consommation simultanée et non séparable. Une section suivante reviendra plus en détails sur la notion de bien commun ${ }^{11}$.

Troisièmement, la troisième catégorie renvoie largement à la notion de bien commun plutôt qu'à celle de bien public. En effet, si la paix ou la santé peuvent être recherchées via des politiques globales, le bénéfice de la paix ou de la santé est inséparable de sa 
production. La paix est consommée de manière inséparable de sa production, tout comme la santé. Il s'agit donc de biens communs.

Il ressort de ces remarques que la distinction à maintenir reste la même que pour les biens publics c'est-à-dire une distinction entre biens publics qui n'ont pas besoin d'être produits-naturels mais qui peuvent faire l'objet d'une dégradation en raison d'externalités, et les biens publics qui nécessitent d'être produits. Mais cette dernière catégorie doit être expurgée des biens communs. Les biens publics mondiaux fabriqués par l'action humaine sont donc des biens non exclusifs et non rivaux dont la production est indépendante -séparable- de la consommation. Il apparaît cependant que cette catégorie de biens est plutôt infime voire utopique. Peut-on penser actuellement à un bien qui bénéficierait potentiellement à tout le monde, ou du moins à une partie non négligeable de la population mondiale, c'est-à-dire qu'il ne serait pas rival, sans que l'on pense aussitôt à la possibilité d'en exclure les bénéficiaires afin de les contraindre à un paiement individualisé. On pourrait penser dans une certaine mesure à des satellites de télécommunication.

Les biens publics mondiaux présentent certes une nouvelle dimension de par la territorialité et la temporalité qu'ils couvrent, mais si on se limite à ces purs aspects géographiques et temporels, la notion ne semble pas apporter grand-chose par rapport aux concepts examinés précédemment. Au contraire elle apporte plutôt confusion. L'originalité de la notion trouve sa pertinence dans la référence implicite à un autre critère que le critère d'efficience, celui d'équité.

\subsection{Un critère d'équité implicite}

40 La notion de bien public mondial, comme elle vient d'être interprétée reste ancrée sur la problématique des conditions d'efficience dans la production ou la préservation. Or il semble que si la catégorisation proposée par Kaul et al. (1999) est ambiguë, c'est en partie parce qu'elle laisse implicite un critère autre que celui d'efficience, celui d'équité.

41 Sandmo (2003) a particulièrement bien mis en lumière la nécessité de tenir compte de ce critère. Il étend le modèle de dépense publique optimale de Samuelson (1954) au niveau international. Il considère deux pays (un pays riche et un pays pauvre) et deux biens (un bien privé et un bien public). Par le biais de la notion de variation d'efficience dans la production du bien public mondial, il suggère que des gains d'efficience pourraient être obtenus par l'exploitation des avantages comparatifs des pays dans la production de ce bien.

42 Si on suppose que la production totale de biens privés est donnée et qu'il faille produire un bien public, avec pour intrant des biens privés. Alors, si la production du bien public est moins coûteuse dans un pays pauvre, celui-ci pourrait, et aurait tout intérêt, à en produire davantage.

43 Cependant, cette spécialisation, conforme à la théorie classique des échanges internationaux, aboutirait à un appauvrissement du pays producteur du bien public, le plus pauvre dans cet exemple. En effet, pour produire ce bien public, le pays pauvre devrait y consacrer une partie de biens privés, dont il est moins doté que le pays riche (puisque plus pauvre). Cette réduction de la consommation de biens privés accroîtrait sa pauvreté, puisque de fait, étant données les caractéristiques du bien public (non rivalité et non exclusion), il ne serait pas en mesure de faire payer ce bien public au pays riche. Pensons par exemple à la contribution à la stabilité climatique nécessitant des biens 
privés pour réduire les externalités provoquant son instabilité. Le pays pauvre verrait donc sa situation empirer.

La question de l'efficience ne peut donc être la seule question posée quand on s'intéresse aux biens publics mondiaux. Il ne fait guère de doute que le critère d'équité entre en ligne de compte. Par exemple, dans le modèle de Sandmo, une meilleure efficience ne pourrait être atteinte qu'à la condition que le pays riche consacre une partie de ses ressources en transfert vers le pays pauvre pour le compenser de son utilisation de biens privés dans la production du bien public. Une telle conclusion incite à penser que les biens publics mondiaux ne peuvent avoir réellement sens que si les pays riches consacrent une part conséquente, non seulement à leur production ou à leur préservation, mais aussi aux transferts internationaux vers les pays pauvres, afin qu'ils s'engagent eux aussi dans la production et la préservation de tels biens.

Nous retrouvons là les conclusions de la section précédente, à savoir que l'efficacité relative de différents régimes de propriété dépend étroitement de la répartition des ressources entre agents, en particulier de l'inégalité des droits. Il n'est alors plus possible d'affirmer la supériorité d'un régime sur l'autre en termes d'efficience. Il devient par contre indispensable d'ouvrir la question des changements dans la répartition des droits à la suite de la mise en œuvre d'un nouveau régime. Plus généralement, il devient indispensable de prendre en compte le critère d'équité en plus de celui d'efficience dans l'analyse des régimes de propriété.

3.Elargir la notion de bien public

La section précédente a examiné l'élargissement proposé par la notion de bien public mondial et aboutit à l'idée que cette notion implique certainement une prise en compte du critère d'équité en plus de celui d'efficience. Or ce critère a été utilisé à plusieurs reprises pour appuyer un dépassement de la notion conventionnelle de bien public. Cette section présente deux élargissements particuliers.

3.1.Du bien public au bien jugé public

Le bien public est défini à partir de caractéristiques relevant de sa consommation (non exclusion et non rivalité). Or une telle approche a fait l'objet d'une critique relative au sens subjectif qui pouvait être donné au mot «public». Certains biens, pouvant être considérés comme publics selon l'approche en termes de caractéristiques, ne sont pas perçus par les agents comme relevant du domaine « public ». En revanche, certains biens exclus de la définition en termes de caractéristiques sont au contraire perçus par les agents comme relevant parfaitement du domaine " public ». La salubrité publique ou les systèmes sanitaires sont typiques de ce genre de situation. Wuyts (1992) et Gasper (2002) relèvent ce fait dans le cas de l'Europe au $19^{\mathrm{e}}$ siècle et l'Afrique du Sud sous l'apartheid. Alors qu'en Europe la salubrité publique était considérée comme relevant du domaine public, concernant aussi bien les riches que les pauvres, notamment afin d'éviter la propagation des maladies, l'Afrique du sud sous l'apartheid est un exemple frappant de délimitation de la salubrité publique à un domaine non public. À la place du développement d'un système sanitaire pour les plus pauvres, l'Afrique du sud de cette période a choisi la relocalisation-délocalisation des populations pauvres (et noires) dans des quartiers périphériques de la ville et a accru le coût des transports afin de préserver les quartiers riches (et blancs) des risques de contagions.

Une telle différence indique que le caractère public d'un bien est avant tout un construit social en fonction de ce qui est perçu ou non comme devant relever du domaine public (Wuyts 1992, Gasper 2002). 

publics mondiaux, un bien peut devenir public sans pour autant qu'il ne présente le caractère pur du bien public défini par les caractéristiques de non exclusion et de non rivalité. Cette approche soulève une question de nature différente des discussions précédentes. Il ne s'agit plus de savoir quel système de droits de propriété est efficace, mais quel système de droits de propriété est équitable. En ce sens, le terme public renvoie à une thématique de l'équité et non de la production du bien.

\subsection{Le triangle de Kaul et Mendoza}

50 Mark Malloch Brown dans l'avant-propos à l'ouvrage de Kaul et al. (2003, p. XVI) souligne: «Il est grand temps que tout le monde se rappelle que, au plan national, nous profitons de biens publics tels que les feux de circulation, la santé publique, les bibliothèques et les systèmes judiciaires, d'où la nécessité pressante de poser la question de savoir quels biens publics mondiaux nous devons produire en commun ${ }^{12}$ en cette période d'ouverture des frontières et d'interpénétration croissante des domaines publics nationaux-et d'ouvrir un débat pleinement participatif sur la manière de répondre à cette question.»

51 L'expression "produire en commun » introduit une certaine ambiguïté. Un bien public est-il ce que les individus décident de produire en commun, ce qui renvoie à la perspective subjective précédente, ou est-il un bien dont les caractéristiques (non exclusion, non rivalité) en font un bien public, mais dont la production ne se fait pas nécessairement en commun, mais qui dans une perspective élargie suppose que la production soit réalisée en commun.

52 Cette seconde interprétation se situe dans la ligne d'une vision élargie du caractère public d'un bien proposé récemment par Kaul et Mendoza (2003) à travers l'aspect triangulaire du caractère public d'un bien.

Le triangle de Kaul et Mendoza (2003, p. 36)

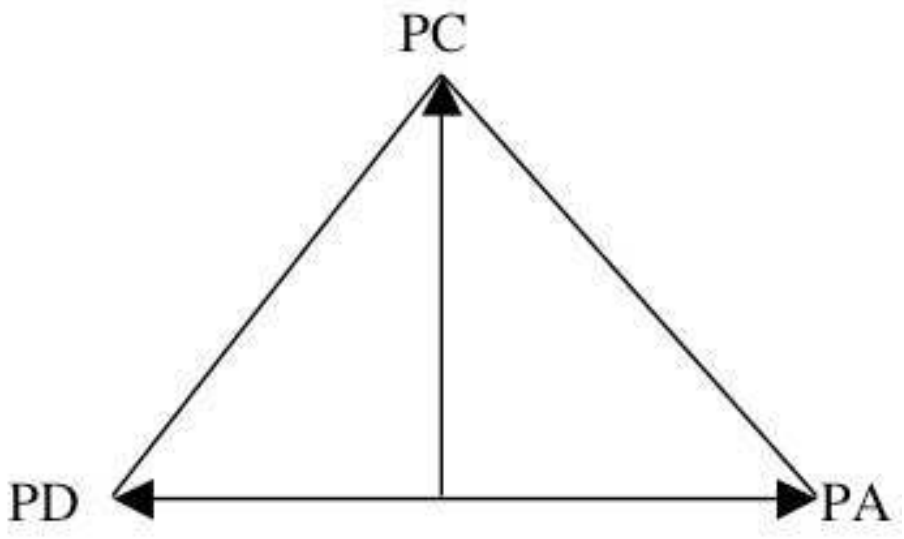

53 Avec PC le caractère public de la consommation, PD le caractère public de la prise de décision et $\mathrm{PA}$ le caractère public de la répartition des avantages. Par rapport au cas idéal, la déformation d'un ou plusieurs côtés du triangle permet d'analyser les distorsions dans le caractère public du bien. Plusieurs exemples peuvent servir d'appui. Ainsi, le régime commercial multilatéral tel qu'il est représenté par l'Organisation Mondiale du Commerce a adopté le principe "un pays-une voix » dans les prises de décisions, mais dans la réalité des distorsions apparaissent du fait des jeux d'influence. Certains pays ont globalement moins d'influence que d'autres (les pays en développement par rapport aux

Développement durable et territoires, Dossier $10 \mid 2008$ 
principaux pays développés) de sorte qu'une distorsion peut apparaître du côté de la répartition des avantages si les décisions reflètent les intérêts des pays dominants. Dans un autre ordre d'idée, la gestion de l'eau est souvent établie au niveau local. Or les effets externes incitent à considérer cette ressource à une échelle plus large (souvent internationale) en raison du caractère public étendu sur la consommation. Une analyse des processus de prise de décision et de la répartition des avantages permet ainsi de caractériser plus finement les enjeux autour de cette ressource.

Les caractéristiques de consommation (non exclusion et non rivalité) permettent toujours de qualifier les biens de biens publics, en revanche les deux autres dimensions ouvrent des perspectives relativisant le caractère public du bien. La dimension prise de décision n'est pas forcément totalement publique, ce qui impliquera probablement que les avantages seront différents selon les agents. La question de l'équité surgit à travers ce schéma puisque une répartition inégale des droits tels les droits de décision peut aussi provoquer des distorsions sur la consommation et la répartition des avantages. Une caractérisation de la notion de " public » à partir de différents niveaux a ainsi l'indéniable mérite de faire valoir la question de l'équité en plus de celle de l'efficacité. Dans une telle optique, un régime de propriété se caractérise au moins par un système de droits possédant les trois dimensions précédentes : décision, consommation, avantages. Tartarin (1987, p. 1138-1139) soulignait : « Il est frappant de voir que, dans la théorie de l'optimum, les ensembles de production et de consommation sont définis formellement avec une grande précision, alors que le système de propriété est à peine évoqué. Les impacts de modifications des préférences, des techniques ou des ressources ont été étudiés tant du point de vue de l'existence de l'équilibre que des variations des grandeurs caractéristiques d'un état initial. Mais rien de comparable n'existe relativement à la structure de propriété. On raisonne pour celle-ci en termes binaires : soit il existe une structure de propriété privée (ou une structure strictement équivalente) et alors on applique les théorèmes fondamentaux, soit la structure de propriété n'est pas assimilable à une structure de propriété privée et alors l'optimum de Pareto n'est pas réalisé. De sorte que l'on se trouve confronté à une liaison très forte entre optimum de Pareto et propriété privée alors même que les formes de propriété n'ont pas été définies avec un degré de précision comparable aux autres déterminants de l'optimum ». Mais on le voit avec le triangle de Kaul et Mendoza, dès lors que le système de propriété est précisé, notamment avec différents niveaux de droits, il devient tout aussi important de tenir compte de l'équité que de l'efficacité. Or la question de l'équité renvoie d'un point de vue purement économique à une double interrogation : tenir compte de ou produire quoi pour qui ? De ce point de vue, un autre concept, celui de « bien commun » permet d'entrevoir certaines pistes de réponses à cette question.

4. Des biens communs au Bien commun

Walzer (1983) relevait que la soumission à l'échange marchand d'un certain nombre de choses, telles l'honneur, la liberté d'expression, la dignité, l'amour, etc., paraissait relativement intolérable, probablement parce que toutes ces choses font partie d'une vision de ce que doit être une société juste. Titmuss (1970), dans un autre registre, celui du don du sang, a montré que le fait de soumettre à l'échange marchand certaines choses provoquait une certaine inefficacité en raison de la répugnance morale de certaines personnes à accepter l'application d'un tel système de droits sur ces choses ${ }^{13}$. L'échange marchand ne semble donc pas pouvoir s'imposer comme seule solution, même si de nombreuses évolutions historiques vont certainement dans le sens d'une plus grande 
emprise du marché sur la vie, parce que le sentiment de partager quelque chose en commun est une dimension importante de la vie. A cet égard, La notion de bien commun s'est développée en parallèle de celle de bien public mondial. Mais elle présente une certaine ambiguïté parce qu'elle peut être lue de deux manières au moins: soit elle renvoie aux caractéristiques des biens en comparaison avec les biens publics (et alors l'utilisation de biens communs est avec un « $b$ » minuscule), soit elle renvoie à la notion de Bien (avec un «B » majuscule) dans le sens de ce qui est bon ou de la vie bonne. Or comme le souligne Ricoeur (1990), la visée éthique de la vie bonne s'inscrit avec et pour les autres dans des institutions justes. La notion de bien commun nous ramène alors inéluctablement au critère d'équité.

\subsection{Des biens communs}

La première analyse que nous pouvons faire de la notion de bien commun concerne les caractéristiques des biens rangés sous ce vocable. Ce qui différencie le bien commun du bien public est que le bien public est produit de manière instrumentale dans une optique d'accroissement du bien-être collectif, parce que laissé à l'action des individus ce type de bien ne serait généralement pas produit ${ }^{14}$, tandis que le bien commun est un bien partagé pour lui-même et qui est le résultat des interactions individuelles (Deneulin et Towsend 2007). Selon Hollenbach (2002) le bien commun est un bien réalisé dans des relations mutuelles dans lesquelles et à travers lesquelles les être humains réalisent leur bien-être. Ainsi, contrairement au bien public, ce n'est pas tellement le bien en lui-même qui produit le bien-être, mais le fait même de le produire en commun. Par exemple, le bienêtre ressenti à jouer de la musique est différent selon que l'on joue seul, à plusieurs, pour un public ou non. Le bien commun n'est donc pas le résultat produit par l'action collective en vue d'accroître le bien-être collectif, c'est le bien partagé lors de cette action collective et concomitant à cette action collective. Selon une telle définition, un bien qui ne nécessiterait pas d'être produit, parce qu'il est librement disponible, ne serait pas un bien commun. Il entrerait dans la catégorie des biens publics naturels ${ }^{15}$.

Par exemple, la musique produite par un orchestre est un bien commun dans le sens où sa production est inséparable du bien en lui-même. La participation à la production du bien, en tant que musicien ou auditeur, permet le bénéfice simultané du bien. Les biens de ce type sont donc inévitablement consommés en commun en tant que produit des interactions individuelles. Au contraire, un bien public a une visée instrumentale clairement séparable des actions des individus. Ainsi, on ne peut pas produire une musique pour orchestre indépendamment de l'action des musiciens, en revanche il est possible de produire un bien public quelle que soit son utilisation, un éclairage public par exemple. Dans ce dernier cas, la production et la consommation sont séparables.

Notons également que le bien commun se distingue d'une externalité. L'externalité renvoie à un effet positif ou négatif produit sur un agent par un autre sans que l'agent affecté par cette externalité ne soit impliqué dans la production de l'effet et sans que l'agent producteur de l'externalité soit lui-même affecté de manière similaire. Le bien commun suppose au contraire qu'il n'y ait pas séparabilité entre production de l'effet et bénéfice ou désagrément causé par l'effet.

Il faut de ce point de vue distinguer le bien commun d'un ensemble d'externalités croisées. Ainsi, la congestion sur la route aux heures d'affluence est le produit simultané de l'ensemble des interactions des individus. Le désagrément causé par la congestion est consommé en même temps qu'il est produit. Mais il s'agit là d'un ensemble d'externalités croisées. La congestion est non-intentionnelle. Au contraire le bien commun est un bien 
produit de manière intentionnelle de sorte que l'agent qui participe à sa production en soit lui-même bénéficiaire. Ainsi, un musicien qui joue de la musique pour des spectateurs bénéficie aussi de cette musique. Et il n'y a guère de doute de ce point de vue que jouer de la musique pour des spectateurs ou jouer de la musique pour soi uniquement sont deux choses différentes.

Mais si le bien commun résulte d'une production intentionnelle par la communauté des individus et non pas les individus eux-mêmes (Dupré 1994), s'ouvre alors la question de quels types de biens la communauté désire produire. Quels types de biens sont désirables ou « valables » pour cette communauté et quels autres types de biens ne le sont pas. Une telle question renvoie à la problématique du Bien et non pas seulement à celle des biens.

\subsection{Vers le Bien commun}

61 La seconde analyse que nous pouvons faire ne concerne plus alors les caractéristiques des biens en eux-mêmes, mais leur contribution à la vie bonne. La notion de Bien commun ne renvoie pas seulement à la promotion du bien-être des individus, autrement dit au bien des individus pris un à un, mais à l'idée du «Bien » dans le sens d'une vie bonne que les individus partagent (Deneulin et Townsend 2007). De ce point de vue, si certains biens communs tels que décrits selon leur caractéristiques peuvent participer au bien commun, on ne peut exclure automatiquement de la vie bonne les biens disponibles librement, indépendamment de l'action humaine comme l'air ou un certain nombre de ressources naturelles. S'ils ne font pas partie de la catégorie des biens communs pris au sens étroit des caractéristiques, ils peuvent être intégrés à une idée du Bien commun, dans le sens qu'ils peuvent contribuer à la vie bonne.

Or si la théorie économique développe sa propre vision du Bien, notamment à travers l'optimum de Pareto, la vie bonne ne peut se réduire à celui-ci (voir par exemple Ballet et Mahieu 2003 pour une première discussion). En particulier, suivant Ricoeur (1990) la vie bonne prend tout son sens avec et pour autrui dans des institutions justes, ce qui nous ramène inévitablement à la question de l'équité.

Si l'équité n'est pas inhérente à la notion de biens communs, elle semble donc pouvoir trouver un ancrage fort dans celle de Bien commun. Mais dans une telle perspective, peut-on échapper à une conception perfectionniste de la justice ${ }^{16}$ ? Quoi qu'il en soit, le perfectionnisme n'est pas incompatible avec le pluralisme. Ross (1930) a par exemple développé une théorie perfectionniste pluraliste tentant de concilier le devoir conséquentialiste de promouvoir le bien et des devoirs non conséquentialistes. Le perfectionnisme a le grand mérite d'insister sur le devoir envers soi-même de poursuivre le Bien, mais aussi de justifier le devoir envers autrui de promouvoir ce même Bien. Le devoir moral consiste certes à rechercher le Bien pour soi mais aussi pour les autres. Une exigence morale d'équité, au-delà de l'efficacité, est donc appuyée par une conception perfectionniste du Bien.

Dans un tel cadre, les biens publics mondiaux deviennent des éléments permettant de favoriser la réalisation du Bien commun. Mais cela suggère que la production et la gestion de tels biens ne reposent pas uniquement sur un critère d'efficience. Un régime de propriété adapté ne doit pas renvoyer uniquement au critère d'efficience mais aussi à celui d'équité. Autrement dit, il n'est plus possible de parler dans un tel cadre de régime de propriété adapté en seule référence à l'efficience (à supposer qu'une telle possibilité puisse exister - voir les critiques présentées dans la première section sur ce point). Un régime de propriété adapté devient un régime qui contribue au Bien commun. Or si celuici ne peut certainement pas mettre de côté l'efficience, il doit aussi tenir compte de 
l'équité. S'ouvre alors les questions, hors de propos de cet article: quelle équité pour le Bien Commun?, et quelle pondération avec l'efficience?

Conclusion

65 L'apparition récente de la notion de biens publics mondiaux est liée à la prise en compte de nouvelles problématiques, notamment dans un contexte de mondialisation, telles que le changement climatique, la paix ou l'insécurité, etc. Cependant, ce concept pris en tant que tel pose problème parce que dans la tradition économique la notion de bien public est définie à partir de deux caractéristiques des biens : la non-rivalité et la non-exclusion. Les biens publics mondiaux renverraient dans une telle optique à des biens possédant ces deux caractéristiques mais dont la temporalité et la territorialité sont étendues par rapport aux précédents. Conformément à la théorie économique conventionnelle ces deux caractéristiques ouvrent une question ardue sur le régime de droits de propriété le plus adapté pour leur production ou leur gestion.

Or les catégorisations des biens publics mondiaux semblent nettement soulever d'autres enjeux que celui de l'efficacité du système de droits de propriété. En particulier, de manière sous-jacente le critère d'équité pointe. Mais la notion de bien public mondial ne laisse que peu entrevoir ce critère.

Le passage de la notion de bien public à celui de bien commun ouvre une autre voie. Dans une conception du Bien commun comme vie bonne, le critère d'équité prend alors tout son sens. Une remise en cause de l'ordre des critères à utiliser pour définir le régime de droits de propriété adapté est alors envisageable. Il semble ainsi préférable de clairement afficher la supériorité d'un critère sur un autre, celui d'équité sur celui d'efficacité plutôt que de recourir à un élargissement des notions laissant penser qu'une telle optique d'équité serait d'emblée prise en compte.

\section{BIBLIOGRAPHIE}

Alchian A.A. (1969), “Corporate Management and Property Rights”, In H. Mane (Ed.), Economic Policy and the Regulation of Corporate Securities, Washington D.C., American Institute for Policy Research.

Alchian A.A. (1987), "Property Rights”, In J. Eatwell, M. Milgrate et P. Newman (Eds), The new Palgrave:A Dictionary in Economics.

Alchian A.A. et Demsetz H. (1972), "Production, Information Costs and Economic Organization", American Economic Review, 62(5): 772-795.

Amann B. (1999), “La théorie des droits de propriété”, In G. Koenig (Ed.), De nouvelles théories pour gérer l'entreprise du XXIème siècle, Paris, Economica.

Anand P.B. (2004), « Financing the provision of global public goods », World Economy, 27(2):

215-237.

Anderson T.L. et Hill P.L. (1975), “The Evolution of Property Rights : A Study of American West”, Journal of Law and Economics, 18(1): 163-178. 
Arrow K.J. (1972), “Gifts and Exchanges”, Philosophy and Public Affairs, 1: 343-362.

Baland J.-M. et Platteau J.-P. (1996), Halting Degradation of Natural Resources : Is There a Role for Rural Communities?, Oxford, FAO and Clarendon Press.

Ballet J. (2000), “Altruisme et biens collectifs: Une revue de la littérature”, Revue Economique, 51 (4) : 789-811.

Ballet J. (2007), "La gestion en commun des ressources naturelles: une perspective critique", Développement durable et territoires, Varia, 29 août, http://revue-ddt.org.

Ballet J. et Mahieu F-R. (2003), Ethique économique, Paris, Ellipses.

Ballet J., Sirven N., Requier-Desjardins M. (2007), "Social Capital and Natural Resource Management: A Critical Perspective”, Journal of Environment and Development, 16(4): 355-374.

Barzel (1989), Economic Analysis of Property Rights, Cambridge, Cambridge University Press.

Benard J. (1985), Economie publique, Paris, Economica.

Boisvert V., Caron A. et Rodary E. (2004), « Privatiser pour conserver ? Petits arrangements de la nouvelle économie des ressources avec la réalité », Tiers Monde, XLI(177) : 61-83.

Breton A. (1964), “The Economics of Nationalism”, Journal of Political Economy, 72: 376-86.

Bromley D.W. (1989), Economic Interests and Institutions, Oxford, Basil Blackwell.

Canto-Sperber M. (sous la dir.) (1996), Dictionnaire d'éthique et de philosophie morale, Paris, PUF.

Cernea M.M. (1987), “Farmer Organisations and Institution Building for Sustainable

Development”, Regional Development Dialogue, 8: 1-24.

Ciriacy-Wantrup S.V. et Bishop R.C. (1975), “Common Property as a Concept in Natural Resources Policy, Natural Resources Journal, 15: 713-727.

Coase R.H. (1937), « The Nature of the Firm », Economica, 4(16): 386-405.

Coase R.H. (1960), “The Problem of Social Cost”, Journal of Law and Economics, 3: 1-44.

Cooper R.N. (1977), « World-Wide vs Regional Integration : is There an Optimal Size of the Integrated Area ?", in F. Machlup (ed), Economic Integration: Worldwide, Regional, Sectoral, New York, Halstead.

Coriat B. et Weinstein O. (1995), Les nouvelles théories de l'entreprise, Paris, Le livre de poche.

Dales J.H. (1968), Pollution, property and Prices, Toronto, University of Toronto Press.

de los Reyes R. et Jopillo S.G. (1986), An Evaluation of the Philippines Participatory Communal Irrigation Program, Quezon City: Institute of Philippine Culture.

Demsetz H. (1967), “Toward a Theory of Property Rights”, American Economic Review, Papers and Proceedings, 57(2): 347-359.

Deneulin S. et Townsend N. (2007), "Public Goods, global public goods and the common good", International Journal of Social Economics, 34(1/2): 19-36.

Dupré L. (1994), “The Common good and the open society”, In Douglas R.B. et Holenbach D. (Eds), Catholicism and Liberalism, Cambridge, Cambridge University Press.

Furubotn E. et Pejovich S. (1972), « Property Rights and Economic Theory : A Survey of Recent Literature », Journal of Economic Literature, 10(4).

Gasper D. (2002), “Fashion, learning and values in public management: reflections on South African and International experience", Africa Development, 27(3/4): 17-47. 
Hardin G.J. (1968), “The Tragedy of Commons”, Science, 162: 1243-1248.

Heltberg R. (2001), “Determinants and Impact of Local Institutions for Common Resource Management”, Environment and Development Economics, 6(2): 183-208.

Heltberg R. (2002), “Property Rights and Natural Resource Management in Developing Countries”, Journal of Economic Surveys, 16(2): 189-214.

Hirshleifer J. (1983), “From Weakest-Link to Best-Shot: The Voluntary provision of Public Goods”, Public Choice, 41: 371-386.

Hollenbach D. (2002), The Common Good and Christian Ethics, Cambridge, Cambridge University Press.

Hurka T. (1996), «Perfectionnisme », in Dictionnaire d'éthique et de philosophie morale, CantoSperber M. (sous la dir.), Paris, PUF : pp.1179-1185.

Kaul I., Grunberg I. et Stern M.A. (1999), Global Public Goods: International Cooperation in the 21st Century, Oxford, Oxford University Press.

Kaul I. Conceiçao P., Le Goulven K., Mendoza R.U. (2003), Fournir des biens publics mondiaux. Gérer la mondialisation, New York, PNUD et Oxford University Press.

Kaul I. et Mendoza R.U. (2003), « promouvoir la notion de bien public », In Kaul I. Conceiçao P., Le Goulven K., Mendoza R.U. (Eds), Fournir des biens publics mondiaux. Gérer la mondialisation, New York, PNUD et Oxford University Press.

Kindleberger C.P. (1986), “International Public Goods without International Government”, American Economic Review, 76(1): 1-13.

Larson B.A. et Bromley D.W. (1990), "Property Rights, Externalities, and Resource Degradation. Locationg the Tragedy", Journal of Development Economics, 33: 235-262.

Liebecap G.D. (1989), Contracting for Property Rights, Cambridge, Cambridge University Press.

Mäler K.G. (1990), “International Environmental Problem”, Oxford Review of Economic Policy, 6(1): 80-108.

Milgrom P. et Roberts J. (1992), Economics, Organization and Management, Londres, Prentice-Hall International.

North D.C. (1990), Institutions, Institutional Change and Economic Performance, Cambridge, Cambridge University Press.

North D.C. et Thomas R.P. (1977), “The First Economic Revolution”, The Economic History Review, 30: 229-241.

Olson M. (1965), The Logic of Collective Action: Public Goods and The Theory of Groups, New York, Schocken Books.

Ostrom E. (1990), Governing the Commons - The Evolution of Institutions for Collective Action. New York: Cambridge University Press.

Pretty J., Thomson J., \& Kiara J.K. (1995), “Agriculture Regeneration in Kenya: the Catchment Approach to Soil and Water Conservation", Ambio, XXIV(1): 113-136.

Ricketts M. (1987), The Economics of Business Enterprise, Brighton, Wheatsheaf Books.

Ricoeur P. (1990), Soi-même comme un autre, Paris, Seuil.

Röling R.N.R. et Wagemakers M.A. (1997), Social Learning for Sustainable Agriculture, Cambridge, Cambridge University Press. 
Ross W.D. (1930), The Right and The Good, Oxford, Clarendon Press.

Runge C.F. et Bromley D.W. (1979), Property Rights and the First Economic Revolution: The Origins of Agriculture Reconsidered, Center for Resource Policy Studies, Working paper $n^{\circ} 13$, Madison, WI, University of Wisconsin.

Samuelson P.A. (1954), « The Pure Theory of Public Expenditure », Review of Economics and Statistics, 39 : 387-389.

Sandler T. (2003), "Evaluer l'offre optimale de biens publics, ou la quête du Saint-Graal », In Kaul I. Conceiçao P., Le Goulven K., Mendoza R.U. (Eds), Fournir des biens publics mondiaux. Gérer la mondialisation, New York, PNUD et Oxford University Press.

Sandmo A. (2003), « Les aspects internationaux de l'offre de biens publics », In Kaul I. Conceiçao P., Le Goulven K., Mendoza R.U. (Eds), Fournir des biens publics mondiaux. Gérer la mondialisation, New York, PNUD et Oxford University Press.

Seabright P. (1993), “Managing Local Commons: Theoretical Issues in Incentive Design”, Journal of Economic Perspectives, 7(4): 113-134.

Serfati C. (2003), «La mondialisation : quelques enjeux problématiques », in C. Serfati (ed), Enjeux de la mondialisation : un regard critique, Toulouse, Octarès.

Singer P. (1973), «Altruism and Commerce : A Defence of Titmuss against Arrow », Philosophy and Public Affairs, 2 : 312-20.

Tartarin R. (1982), “La théorie des droits de propriété: vers un historicisme libéral, in L'économiefiction contre les nouveaux économistes, Paris, Maspero, coll. Textes à l'appui, pp.105-135.

Tartarin R. (1987), « Efficacité et propriété », Revue Economique, 6 : 1129-1156.

Titmuss R.M. (1970), The Gift Relationship, Allen \& Unwin.

Uphoff N. (1992), Learning from Gal Oya: Possibilities for Participatory Development and Post-Newtonian Science, Ithica: Cornell University Press.

Wade R. (1988), Village Republics: Economic Conditions for Collective Action in South India, Cambridge: Cambridge University Press.

Walzer M. (1983), Spheres of Justice, New York, Basic Books.

Wuyts M. (1992), “Deprivation and Public need”, In Wuyts M., Mackintosh M. et Hewitt T. (Eds), Development policy and Public Action, Cambridge M.A., Harvard University Press.

\section{NOTES}

1. Cet article a été présenté lors de la journée "Biens communs et propriété" organisée le 10 mai 2007 à l'université de Lille 1. Les remarques des participants à la journée ont contribué à son évolution. Je remercie aussi vivement Bruno Boidin et Sandrine Rousseau pour m'avoir incité à travailler sur ce texte. Je remercie enfin très vivement Olivier Petit pour ses très nombreuses remarques sur une version antérieure.

2. Une telle approche renvoie plus généralement à l'idée de marché contingent. Un système de marché dont l'efficience est remise en cause par une externalité peut retrouver son efficience si un nouveau marché est créé pour répondre à cette externalité. 3. Notons ici que cette appellation est problématique puisque le terme " communaux » laisse penser que le problème est relatif au régime de propriété en commun alors qu'il est 
relatif à celui de l'accès libre. Il s'agit là d'une confusion dont Hardin est à l'origine (Ciriacy-Wantrup et Bishop 1975).

4. La théorie des droits de propriété a connu des développements dans le cadre de système de droits incomplet. Considérant que tout système de droits de propriété est par nature incomplet les développements ultérieurs de la théorie des droits de propriété mettent l'accent sur deux conditions d'efficacité des systèmes de droits : l'allocation du rendement résiduel et la détention du contrôle résiduel (Milgrom et Roberts 1992). En effet, la production d'un bien public est confrontée au problème de l'action collective tel que soulevé par Olson (1965). Le comportement de passager clandestin est par conséquent la règle. Or comme le soulignent Alchian et Demsetz (1972), un tel comportement peut être évité si un agent jouant le rôle de «moniteur » (contrôleur) des actions des autres est institué. Il faut que cet agent soit clairement distinct des autres, ait un statut différent, sinon il serait lui-même enclin à adopter un comportement de passager clandestin. Cet agent doit donc avoir un statut spécifique, correspondant à une structure de droits de propriété originale, et plus particulièrement ce statut doit refléter cinq caractéristiques du système de droits de propriété (Coriat et Weinsten 1995) :1) Il doit être le créancier résiduel, 2) il doit avoir le droit d'observer et contrôler le comportement des détenteurs de ressources participants à la production, 3) il doit posséder le droit exclusif à être dans un rapport contractuel avec tous les détenteurs de ressources, 4) il doit posséder le droit de modifier la composition des participants à la production, 5) il doit avoir le droit de vendre le statut particulier qu'il détient. Il faut souligner ici que si, en théorie, n'importe quel agent peut être moniteur, ce sont souvent les autorités publiques qui jouent ce rôle. Ainsi, paradoxalement, lorsque le système de droits de propriété est incomplet l'Etat devient souvent le garant de son bon fonctionnement, de sorte qu'un système de droits de propriété privée sans cette garantie serait souvent inefficient.

5. Notons ici avec Boisvert et al. (2004) que les marchés de droits à polluer proposés par John Dales (1968) ont été assimilés à une privatisation des biens environnementaux, alors qu'il s'agit là d'une interprétation abusive et loin du mécanisme proposé par Dales luimême. Pour une discussion détaillée voir Boisvert et al. (2004).

6. Si on peut considérer que les biens publics ont en général une probabilité élevée de tomber sous le régime d'accès libre, il n'y a évidemment rien d'automatique dans la mesure où tout dépend du comportement des individus et des normes sociales plus ou moins respectées en vigueur. Ainsi, un bien public pourrait ne connaître aucune dégradation si les individus adoptent des comportements qui de fait reviennent à former un régime de propriété commune, dont on peut penser par exemple que les normes sociales jouent un rôle de contrôle fort.

7. Notons que même si on relâche l'hypothèse d'égoïsme des agents économiques et que l'on postule un altruisme généralisé, la fourniture du bien public est généralement sous optimale. Pour une présentation de ce problème, voir par exemple Ballet (2000).

8. Notons toutefois que la question de la production d'un bien public ne se résume pas à celle du régime de droits de propriété. Pour une analyse plus complexe voir par exemple la distinction de Hirshleifer (1983) entre les trois technologies de production alternatives suivantes : la sommation, le maillon faible, la contribution du meilleur. Pour une présentation et discussion voir Sandler (2003).

9. Tartarin (1987) souligne que les partisans de la théorie des droits de propriété ont souvent tenté de minorer l'importance des biens publics.

10. Pour une présentation nuancée de la gestion en commun des ressources voir aussi Ballet (2007) et Ballet et al. (2007). 
11. Notons cependant dès à présent que la notion de bien commun est elle-même une source d'ambiguïté puisqu'il peut s'agir soit d'un bien au sens économique du terme, soit d'un bien en tant qu'antonyme du mal.

12. C'est nous qui soulignons.

13. La thèse de Titmuss a provoqué un bon nombre de réactions, dont en particulier Arrow (1972) et Singer (1973).

14. Nous utilisons bien sûr ici la notion de bien public au sens le plus usuel de la théorie économique c'est-à-dire en relation uniquement avec les caractéristiques de cette catégorie de bien. Dans un tel cadre, l'accroissement de bien-être ne correspond qu'à un état Pareto supérieur sans référence à la distribution des revenus.

15. On voit donc tout de suite l'ambiguïté de cette notion de bien commun car on peut penser que certaines ressources naturelles constituent un bien que nous partageons en commun.

16. Rappelons qu'une théorie morale perfectionniste se caractérise par une visée théorique du bien humain. Le perfectionnisme commande en particulier aux agents de choisir les actions qui favoriseront le plus ce qui est bien. Ce qui est bien n'est pas identique ici la notion de bonheur. Pour une présentation du perfectionnisme, voir par exemple l'article de Thomas Hurka dans le dictionnaire d'éthique et de philosophie morale, sous la direction de Monique Canto-Sperber, Paris, PUF, 1996.

\section{RÉSUMÉS}

La notion de bien public mondial s'est développée ces dernières années. Elle renvoie à un dépassement ambigu de la notion usuelle dans la théorie économique de bien public. A cette occasion elle ouvre le débat sur l'équité en complément de l'efficience. De ce fait, la confrontation de cette nouvelle notion avec l'analyse de l'efficience des régimes de propriété s'impose. Par ailleurs, la confrontation avec la notion de bien commun, notion elle aussi en développement, éclaire l'enjeu autour de l'équité.

Property, Global Public Goods, Common Good(s) : A Lecture. The concept of global public good emerged these last years. It proposes, in an ambiguous way, to go beyond the usual concept of public good. Then, added to usual questions on efficiency, the debate on fairness becomes a stake. Confrontation with analysis on property rights command attention, Furthermore, confrontation with the concept of common good, also a developing concept, highlight stake around fairness.

\section{INDEX}

Mots-clés : biens publics, bien commun, propriété, bien public mondial

Keywords : Global public good, Public good, Property right, Common good 


\section{AUTEUR}

\section{JÉRÔME BALLET}

Université de Versailles Saint Quentin en Yvelines, UMR 063 C3ED (IRD-UVSQ, 47 Bd Vauban, 78047 Guyancourt cedex, France. 